\title{
Ex Vivo Scanning Electron Microscopy Evaluation of Cleaning Efficacy Following In Vivo Endodontic Treatment: A Report of 2 Cases
}

\section{Enrico E DiVito* and Shahriar A Rassoulian}

Arizona Center for Laser Dentistry, Scottsdale, Arizona, USA

*Corresponding authors: Enrico E. DiVito, DDS, PC. Arizona Center for Laser Dentistry, 7900 E. Thompson Peak Parkway \#101, Scottsdale, AZ 85255, USA, Tel: +4809901905; Fax: 4809902311; E-mail: divitodds@gmail.com

Received date: March 09, 2017; Accepted date: March 15, 2017; Published date: March 22, 2017

Copyright: ( 2017 DiVito EE, et al. This is an open-access article distributed under the terms of the Creative Commons Attribution License, which permits unrestricted use, distribution, and reproduction in any medium, provided the original author and source are credited.

\begin{abstract}
In endodontic therapy, thorough cleaning of the root canal system which results in the complete or near elimination of diseased or necrotic pulp tissue and resident microorganisms is the most critical factor in ensuring a favorable outcome. Removal of both debris and the smear layer from the canals is important, since organic matter from these materials may promote bacterial growth and inhibit obturation materials from having proper contact with the canal wall. This report of two cases is the first known presentation of teeth receiving in vivo treatment and then analyzed post extraction via ex vivo scanning electron microscopy (SEM).

The two cases describe patients with similar clinical presentation and diagnoses who required single-tooth extractions. The treating clinician provided in vivo chairside endodontic therapy using two different available treatment modalities: standard root canal treatment and the GentleWave $₫$ Procedure. The teeth were then extracted and underwent ex vivo SEM analyses as part of an ongoing clinical study. Similarities and differences in the level of debridement in the root canal systems were observed between the two cases, with greater reduction in accumulated debris and smear layer scores present in the tooth treated with the GentleWave Procedure. Further research is ongoing to provide additional evidence-based data regarding outcomes related to the cleaning efficacy of different endodontic therapies.
\end{abstract}

Keywords: Root canal therapy; ex vivo; GentleWave; Multisonic UItracleaning "'; Scanning electron microscopy

\section{Introduction}

Successful endodontic treatment is reliant on the establishment of an accurate diagnosis, proficient knowledge of tooth anatomy and morphology, and the effective cleaning, shaping, and debridement of the root canal system $[1,2]$. Thorough cleaning that results in the complete or near elimination of diseased or necrotic pulp tissue and resident microorganisms is the most critical factor in ensuring a favorable outcome $[1,2]$.

Standard root canal therapy (RCT) can be challenged by patientspecific morphologic factors such as lateral and accessory canals, canal wall irregularities and isthmuses [2,3], which can make total debridement of the canals difficult and potentially hinder the clinical outcome. Past research has shown that standard instruments and rotary files may not completely debride the canals $[4,5]$ or significantly remove the smear layer prior to obturation [6,7]. Removal of both debris and the smear layer from the canals is important, since organic matter from these materials may promote bacterial growth and inhibit obturation materials from having proper contact with the canal walls $[2,8,9]$.

Although there are a number of techniques and instrumentation protocols for treating the vast variety of clinical scenarios, there is no current consensus on which technique or instrument is superior $[2,5]$. Past meta-analyses of endodontic treatment studies have reported success rates of $86 \%$ with standard methods [10], and a reported success rate of $97 \%$ for the newer GentleWave ${ }^{\circledR}$ System [11].
This report of two cases describes patients of similar clinical presentation and diagnoses who required single-tooth extractions. Endodontic therapy was provided by the same clinician using two different treatment modalities: standard RCT and the GentleWave Procedure. The teeth were then extracted and underwent ex vivo scanning electron microscopy (SEM) analyses as part of an ongoing clinical study. This is the first known presentation of in vivo treated teeth analyzed post extraction via ex vivo SEM.

\section{Case Presentation \# 1}

A 66-year-old female presented for examination at a general dentist's office. After clinical examination, it was considered that the only remaining maxillary molar (\#15) undergo extraction. The patient was informed of various modalities of possible treatment and declined to attempt to salvage the remaining maxillary tooth. The patient requested tooth extraction and complete maxillary dentures, and was then referred for possible inclusion into a clinical study evaluation at an endodontic practice.

The treating endodontist reviewed the patient's medical and dental history and deemed it non-contributory and unremarkable. Clinical examination of the molar showed mild periodontitis with some bleeding and no painful response to percussion or palpation.

Vitality testing with Endo-Ice (Coltene $^{\varpi}$ /Whaledent, Cuyahoga Falls, $\mathrm{OH}$ ) revealed a normal response. Based on clinical and radiographic findings (Figure 1), a diagnosis of normal pulp and periradicular tissue was made. The clinical study treatment plan was reviewed with the patient, whereby the subject tooth undergoes RCT 
followed by extraction and ex vivo analyses. The patient agreed and informed consent was obtained.

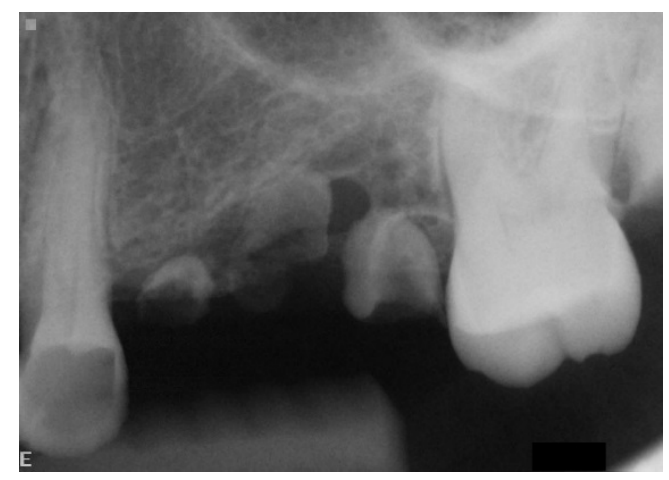

Figure 1: Tooth \#15-Radiograph Prior to Standard Root Canal Therapy.

The patient received local anesthesia per standard techniques, and a dental dam was applied to isolate the tooth for endodontic treatment. Endodontic access was obtained, a carious lesion was removed and the missing tooth structure restored. Examination of the pulp chamber floor revealed four distinct canals: palatal, distobuccal, mesiobuccal-1 and mesiobuccal-2. All canals were shaped to a depth of $5 \mathrm{~mm}$ into each canal using Gates Glidden Drills (Kerr" then each orifice using size \#3. Patency was gained with a \#15 K-file, and working length was established using a Root ZX apex locator (J. Morita, Irvine, CA). Calcification was present throughout the mesiobuccal-2 canal and bleeding occurred during the shaping process. All canals were instrumented using K-files up to \#20 and ProTaper $^{\bullet}$ rotary files to F2 (Dentsply, Tulsa, OK). Irrigation was performed with $6.0 \%$ sodium hypochlorite $(\mathrm{NaOCl})$ followed by $17 \%$ ethylenediaminetetraacetic acid (EDTA), and a final rinse of chlorhexidine. The root canals were dried with paper points and the canal orifices were sealed with cotton, followed by a layer of Cavit ${ }^{\mathrm{Tu}}$ (3M, Neuss, Germany) and then composite. At the conclusion of the $\mathrm{RCT}$, the tooth was extracted for further ex vivo analyses per the study protocol. Post-extraction, the tooth was rinsed in a dental sink under running water to remove any saliva and blood. The tooth was then placed in 1X phosphate-buffered saline (PBS) solution $\mathrm{pH} 7.4$ (Thermo Fisher Scientific, Fair Lawn, NJ) and transported for ex vivo analyses. Post-treatment follow-up of the patient was conducted 48 hours after the procedure, and the patient reported no complications or discomfort.

\section{Case Presentation \#2}

A 27-year-old female presented for examination at a general dentist's office with a chief complaint of spontaneous pain. After clinical examination, it was determined that a right maxillary molar (\#2) was unrestorable. Single-tooth extraction was recommended and the patient was referred for possible inclusion into a clinical study evaluation at an endodontic practice.

The treating endodontist reviewed the patient's medical and dental history and deemed it non-contributory and unremarkable. Upon clinical examination, the tooth revealed a deep carious lesion with pulpal exposure which had been excavated and filled with temporary filling material. Probing indicated mild periodontitis. Periradicular testing elicited a mild painful response to palpation and no sensitivity to percussion. Vitality testing with Endo-Ice produced a moderate painful lingering response. Based on clinical and radiographic findings (Figure 2), a diagnosis of irreversible pulpitis with asymptomatic apical periodontitis was made. The clinical study treatment plan was reviewed with the patient, whereby the subject tooth undergoes RCT with the GentleWave system, followed by extraction and ex vivo analyses. The patient agreed and informed consent was obtained.

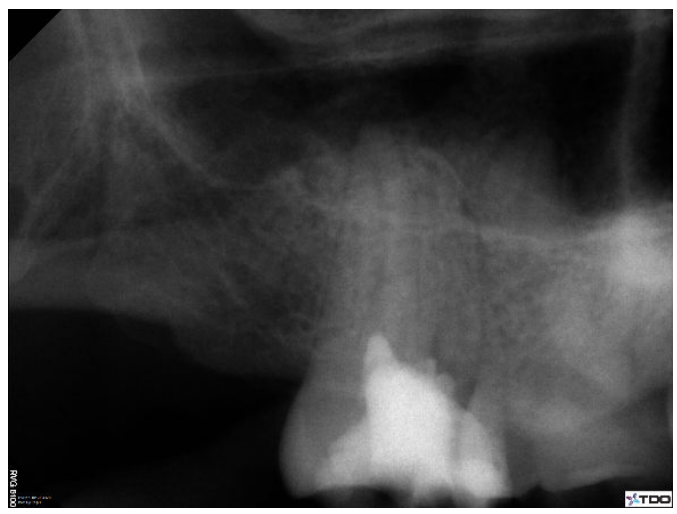

Figure 2: Tooth \#2-Radiograph Prior to GentleWave Procedure.

The patient was anesthetized utilizing standard dental techniques and the tooth was isolated with a dental dam. The carious lesion was removed and the missing tooth structure was restored. A conservative straight line access opening was prepared with removal of all pulp horns and ledges. Examination of the pulp chamber floor revealed four distinct canals: palatal, distobuccal, mesiobuccal-1 and mesiobuccal-2. To preserve tooth structure, orifice openers or Gates Glidden burs were not utilized during the instrumentation process. Patency was gained with a K-file type \#15 and working length was established using a Root ZX apex locator (J. Morita, Irvine, CA). EndoSequence (Brassler $\mathrm{USA}^{\circ}$, Savannah, GA) rotary files $15 / .04$ were utilized to create a fluid path prior to the GentleWave Procedure (Sonendo, Laguna Hills, CA). Bleeding and pain occurred during the instrumentation process, and the pulp appeared severely hyperemic within the palatal canal. A temporary platform was created to maintain a sealed environment for optimum MultiSonic UltraCleaning ${ }^{\text {ma }}$ during the GentleWave Procedure. Post GentleWave Procedure, an uninstrumented mesial isthmus was visualized. The root canals were dried with paper points and the canal orifices were sealed with cotton, followed by a layer of Cavit $^{\text {mi }}$ (3M, Neuss, Germany) and then composite material. At the conclusion of the root canal cleaning, the tooth was extracted for further ex vivo analyses per the study protocol. Post-extraction, the tooth was rinsed in a dental sink under running water to remove any saliva and blood. The tooth was then placed in $1 \mathrm{X}$ phosphate-buffered saline (PBS) solution pH 7.4 (Thermo Fisher Scientific, Fair Lawn, NJ) and transported for ex vivo analyses. Post-treatment follow-up was conducted 48 hours after the procedure, and the patient reported no complications or discomfort.

\section{Ex Vivo Analyses}

Both teeth were prepared for ex vivo analyses in the following manner: A diamond disc was used to score a groove around the entire circumference of the cementoenamel junction, short of breaching the pulp chamber. Air and light water spray was used to remove superficial 
debris from the external surface of the tooth. A \#15 blade was inserted into the score marks, and light successive taps with a hammer were used to separate the crown from the roots. To separate the roots, the diamond disc was used to score in-between the roots, without breaching the pulp chamber or canals. Then the blade was inserted into the score marks, and light successive taps with a hammer were used to separate the roots. The roots were then split using the diamond disc to score along the coronal/apical axis of the root, without breaching the canal.

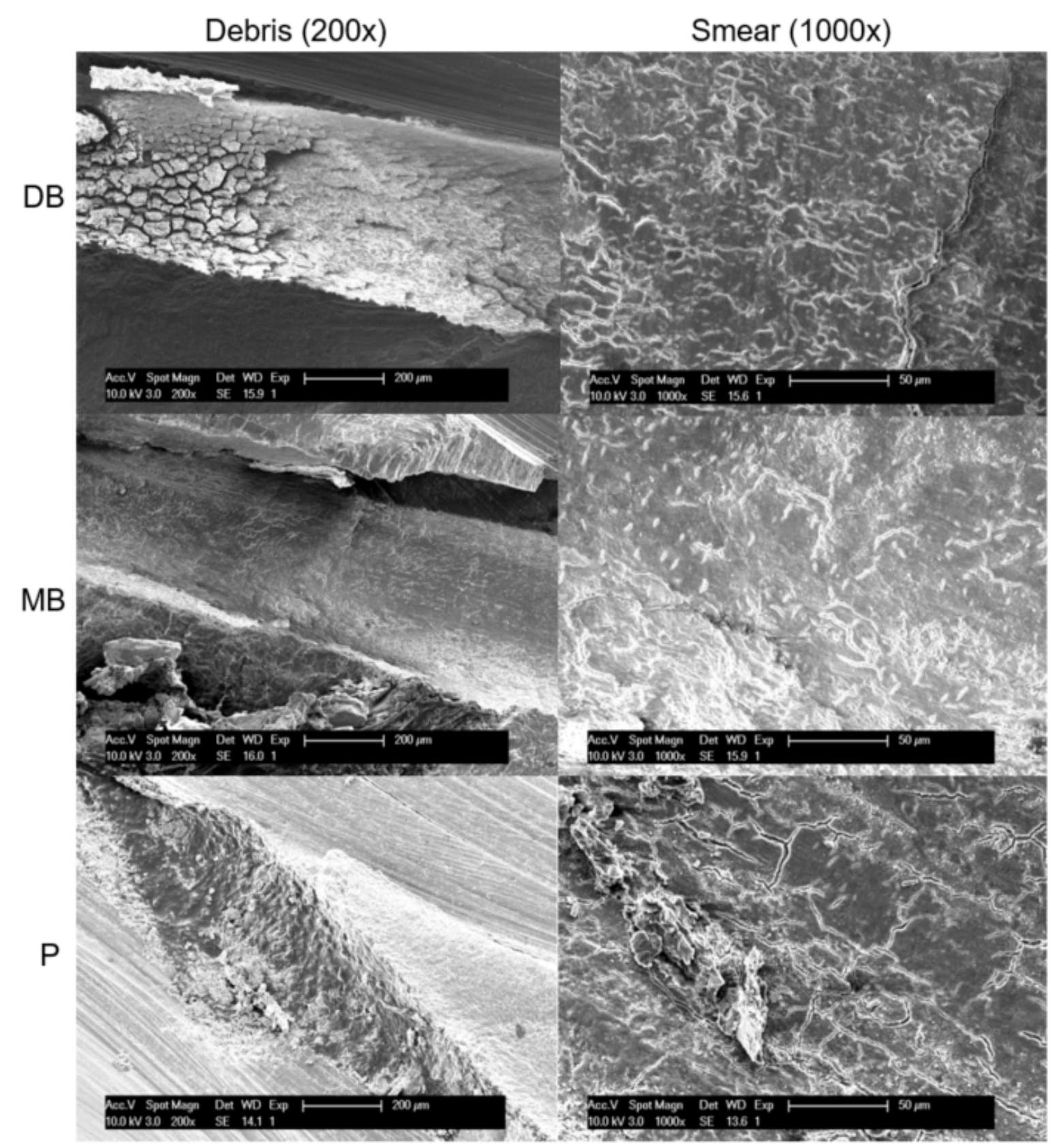

Figure 3: SEM Micrographs of the root canal wall after use of standard root canal therapy. Images obtained with SEM at different magnification levels (DB: Distobuccal surface; MB: Mesiobuccal surface; P: Palatal surface).

The tooth halves were subjected to increasing concentrations of ethanol $(50 \%, 70 \%, 80 \%$, and $100 \%)$ for serial dehydration. After the final rinse, the surface of the root canal wall in each tooth was imaged using SEM (Phillips XL30 FEG, FEI, Hillsboro, OR).
The root sections were observed using a standardized method per Bechelli et al. [12]. Micrographs were taken of the root sections at 200X magnification for debris analysis and 1000X magnification for smear layer analysis (Figures 3 and 4). Levels of debris and smear layer removal were scored using the classification methods of Gutmann et al 
Citation: DiVito EE, Rassoulian SA (2017) Ex Vivo Scanning Electron Microscopy Evaluation of Cleaning Efficacy Following In Vivo Endodontic Treatment: A Report of 2 Cases. Dentistry 7: 419. doi:10.4172/2161-1122.1000419

Page 4 of 6

[13]. A score of 1-4 was assigned to each root surface to reflect debris load and presence of residual smear layer. treated canals, though the patient treated with the GentleWave System had markedly less residual debris and smear layer (Tables 1 and 2).

Images from the SEM analyses revealed that both treatments achieved significant reduction of debris and smear layer within the

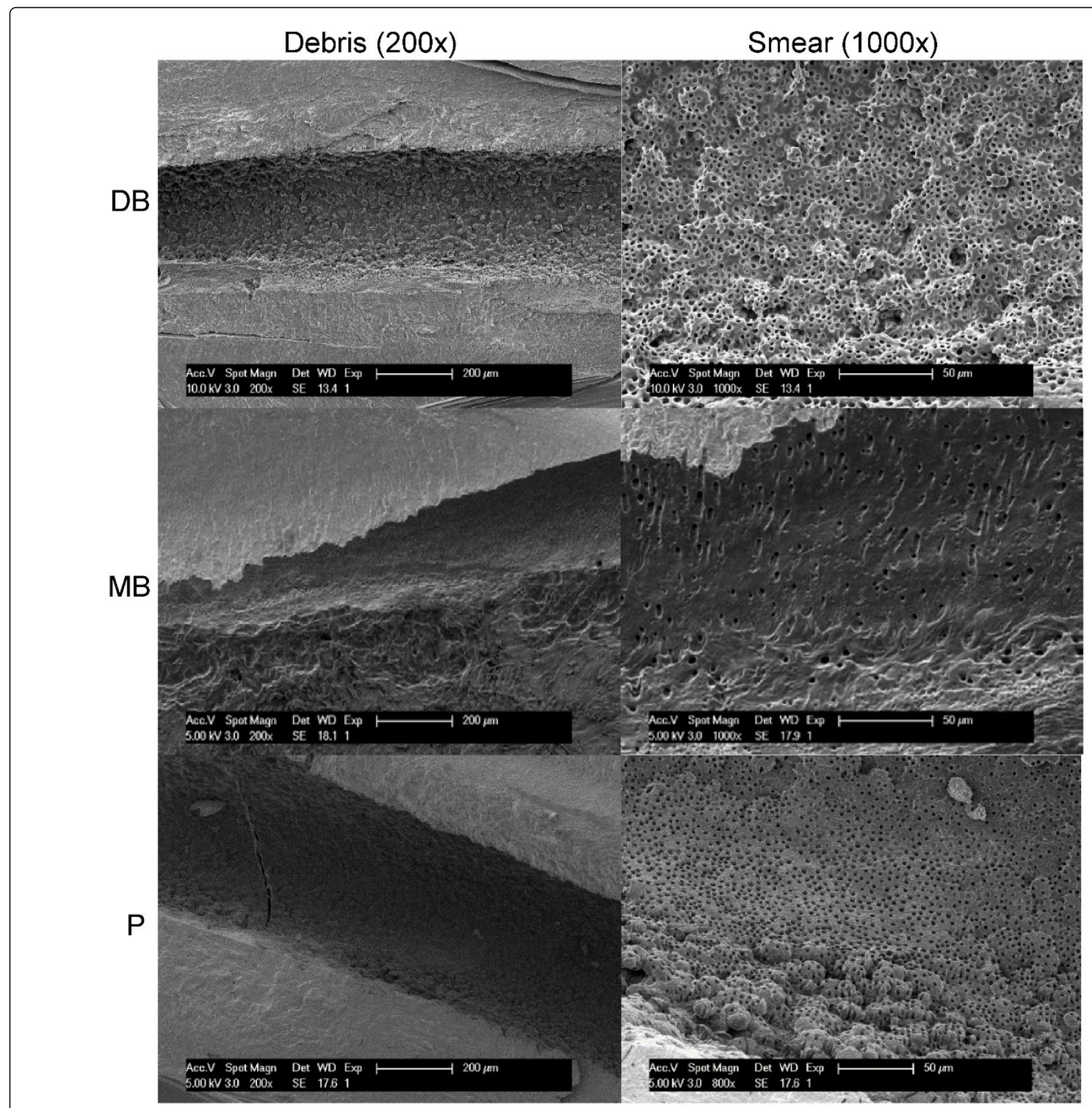

Figure 4: SEM Micrographs of the root canal wall after use of the GentleWave Procedure. Images obtained with SEM at different magnification levels (DB: Distobuccal surface; MB: Mesiobuccal surface; P: Palatal surface).

\section{Discussion}

The present case studies reveal the outcomes of two patients with similar diagnoses, treated by the same clinician but receiving two different endodontic treatment methods. The unusual aspect of these case reports is that the patients received in vivo endodontic therapy chairside, but the teeth were then extracted post-treatment. 


\begin{tabular}{|c|c|c|c|c|c|c|}
\hline \multirow[t]{2}{*}{ Root } & \multicolumn{2}{|l|}{ Apical } & \multicolumn{2}{|l|}{ Middle } & \multicolumn{2}{|c|}{ Coronal } \\
\hline & Debris & Smear & Debris & Smear & Debris & Smear \\
\hline DB & 2.5 & 4 & 2 & 2.5 & 1.5 & 1 \\
\hline MB1 & 3 & 4 & 1 & 3.5 & 1 & 1 \\
\hline MB2 & 3 & 4 & 1 & 1.7 & 1 & 1 \\
\hline $\mathbf{P}$ & 3 & 4 & 1 & 4 & 1.5 & 2 \\
\hline Mean Score & 2.9 & 4.0 & 1.3 & 2.9 & 1.3 & 1.3 \\
\hline
\end{tabular}

Table 1: SEM Scores ${ }^{*}$ Indicating level of residual debris and smear layer post-treatment: Case \# 1 (Standard root canal treatment).

\begin{tabular}{|c|c|c|c|c|c|c|}
\hline \multirow[t]{2}{*}{ Root } & \multicolumn{2}{|l|}{ Apical } & \multicolumn{2}{|l|}{ Middle } & \multicolumn{2}{|c|}{ Coronal } \\
\hline & Debris & Smear & Debris & Smear & Debris & Smear \\
\hline DB & 1 & $N A^{\ddagger}$ & 1 & 1 & 1 & 1 \\
\hline MB1 & 1 & 1 & 1 & 1 & 1 & 1 \\
\hline MB2 & 1 & $N A^{\ddagger}$ & 1 & 1 & 1 & 1 \\
\hline $\mathbf{P}$ & 1 & 1 & 1 & 1 & 1 & 1 \\
\hline Mean Score & 1 & 1 & 1 & 1 & 1 & 1 \\
\hline
\end{tabular}

Table 2: SEM Scores* indicating level of residual debris and smear layer post-treatment: Case \#2 (GentleWave Procedure).

This allowed for exploratory ex vivo examination of the teeth, including a thorough evaluation of the cleaning efficacy of the two root canal cleaning methods.

Although mechanical cleaning is considered the most important aspect of conventional RCT, residual biofilm and debris left in the canals can compromise patient outcomes [14,15]. Standard RCT cannot always reach throughout the complex and irregular anatomies that make up a root canal system [16], which has prompted the development of other root canal cleaning therapies, including the GentleWave System. These novel devices on the endodontic market have been shown to demonstrate improved debridement efficacy in the canals [17-20].

After a patient receives RCT, it can be difficult to thoroughly gauge the level of debridement achieved in the post-treatment setting, unless an obvious clinical event such as infection or persistent discomfort brings the patient back to the treating clinician [21]. In the clinical scenarios described herein, we report on a unique presentation of odontogenic therapies in human subjects, where there was immediate evaluation of the efficacy of treatments through ex vivo analyses of the treated teeth. This is the first known presentation of endodontically treated teeth analyzed in this manner.

Because the treating clinician was the same for both cases, and brought the same skillset and level of experience to each procedure, one can presume that any differences in the findings are due to the treatments themselves and are not skewed by operator variability. The case reports reveal a number of findings; the therapeutic treatment options both achieved reduction of debris and smear layer within the treated canals. However, SEM analyses conducted ex vivo showed that the patient treated with the GentleWave System had notably less debris load and residual smear layer. Although it would be speculative to compare the results between the two cases, the results suggest that the cleaning process was significantly more complete with the GentleWave Procedure as compared to standard RCT. This may be explained by a number of factors, including the presence of less debris from the instrumentation needed with the GentleWave System and its ability to achieve a more rapid tissue dissolution rate and ultimately reduction in bioload $[17,20]$. Further research is warranted.

\section{Conclusion}

In these case reports, the treating clinician provided in vivo endodontic therapy to two patients using a different treatment modality per patient. Extractions and ex vivo analyses were then performed and similarities and differences in the level of debridement in the root canal systems were observed between the two cases. Accumulated debris and residual smear layer scores were substantially lower in the patient treated with the GentleWave Procedure, though caution is warranted in making comparisons between the two cases in this exploratory exercise. Further research is ongoing to provide additional evidence-based data regarding emerging therapeutic options for the endodontic field. 


\section{Acknowledgements}

The clinical study is funded by Sonendo Inc. The authors acknowledge the participation of Dr. Shahriar A. Rassoulian as a Principal Investigator in the clinical study. The authors would like to thank Kathleen Pillsbury Hopf for medical writing support.

\section{Disclosure}

Dr. Enrico E. DiVito is a consultant for Sonendo Inc.

\section{References}

1. Sabeti MA, Nekofar M, Motahhary P, Ghandi M, Simon JH (2006) Healing of apical periodontitis after endodontic treatment with and without obturation in dogs. J Endod 32: 628-633.

2. Johnson WT, Noblett WC (2009) Cleaning and Shaping in Endodontics: Principles and Practice. 4th ed. Saunders, Philadelphia, PA.

3. Peters OA (2004) Current challenges and concepts in the preparation of root canal systems: a review. J Endod 30: 559-567.

4. Shuping GB, Orstavik D, Sigurdsson A, Trope M (2000) Reduction of intracanal bacteria using nickel-titanium rotary instrumentation and various medications. J Endod 26: 751-755.

5. Dalton BC, Orstavik D, Phillips C, Pettiette M, Trope M (1998) Bacterial reduction with nickel-titanium rotary instrumentation. J Endod 24: 763-767.

6. Clark-Holke D, Drake D, Walton R, Rivera E, Guthmiller JM (2003) Bacterial penetration through canals of endodontically treated teeth in the presence or absence of the smear layer. Journal of Dentistry 31: 275-281.

7. Torabinejad M, Handysides R, Khademi AA, Bakland LK (2002) Clinical implications of the smear layer in endodontics: a review. Oral Surgery Oral Medicine Oral Pathology Oral Radiology \& Endodontics 94: 658-666.
8. Oksan T, Aktener BO, Sen BH, Tezel H (1993) The penetration of root canal sealers into dentinal tubules. A scanning electron microscopic study. Int Endod J 26:301-305.

9. Wennberg A, Orstavik D (1990) Adhesion of root canal sealers to bovine dentine and gutta-percha. Int Endod J 23: 13-19.

10. Elemam RF, Pretty I (2011) Comparison of the Success Rate of Endodontic Treatment and Implant Treatment. ISRN Dentistry: 640509.

11. Sigurdsson A, Garland RW, Le KT, Woo SM (2016) 12-month Healing Rates after Endodontic Therapy Using the Novel GentleWave System: A Prospective Multicenter Clinical Study. J Endod 42: 1040-1048.

12. Bechelli C, Zecchi Orlandini S, Colafranceschi M (1999) Scanning electron microscope study on the efficacy of root canal wall debridement of hand versus Lightspeed instrumentation. Int Endod J 32: 484-493.

13. Gutmann JL, Saunders WP, Nguyen L, Guo IY, Saunders EM (1994) Ultrasonic root-end preparation. Part 1. SEM analysis. Int Endod J 27: 318-324.

14. Haapasalo M, Endal U, Zandi H, Coil JM (2005) Eradication of endodontic infection by instrumentation and irrigation solutions. Endodontic Topics 10: 77-102.

15. Haapasalo M, Shen Y (2010) Current therapeutic options for endodontic biofilms. Endodontic Topics 22: 79-98.

16. http://sonendo.com/technology. Accessed February 23, 2017.

17. Haapasalo M, Wang Z, Shen Y, Curtis A, Patel P, et al. (2014) Tissue dissolution by a novel multisonic ultracleaning system and sodium hypochlorite. J Endod 40: 1178-1181.

18. Molina B, Glickman G, Vandrangi P, Khakpour M (2015) Evaluation of Root Canal Debridement of Human Molars Using the GentleWave System. J Endod 41: 1701-1705.

19. Vandrangi P (2016) Evaluating Penetration Depth of Treatment Fluids into Dentinal Tubules Using the GentleWave ${ }^{\circledR}$ System. Dentistry 6: 366.

20. Vandrangi P, Basrani B (2015) Multisonic Ultracleaning ${ }^{\text {int }}$ in Molars with the GentleWave ${ }^{\mathrm{mx}}$ System. Oral Health: 72-86.

21. Zehnder M, Belibasakis GN (2015) On the dynamics of root canal infections-what we understand and what we don't. Virulence 6: 216-222. 